\title{
ÉPOCA E PRINCÍPIOS ATIVOS PARA A QUEBRA DE DORMÊNCIA DE CAQUIZEIRO CV. FUYU ${ }^{1}$
}

\author{
RICARDO ANTONIO AYUB², JULIUS BLUM³ ${ }^{3}$ MARCELO BARBOSA MALGARIM ${ }^{4}$
}

RESUMO- Este trabalho foi realizado com o objetivo de aumentar o período de oferta do caqui 'Fuyu' e buscar alternativas à falta de opções para estimular a brotação, avaliando-se épocas e diferentes princípios ativos na quebra de dormência dos frutos. Os tratamentos testados foram: óleo mineral (OM), cianamida hidrogenada $(\mathrm{CH})$ e a mistura de macro e micronutrientes, em três épocas de aplicação, constituindo-se esquema fatorial com 5 tratamentos e 3 épocas de aplicação. Os tratamentos testados foram: T1-testemunha; T2- 0,5\% OM; T3-: $2 \% \mathrm{CH}+0,5 \% \mathrm{OM}$; T4- $1 \%$ micronutrientes $+0,2 \%$ macronutrientes $+0,5 \% \mathrm{OM}$; T5- $2 \%$ micronutrientes $+0,2 \%$ macronutrientes $+0,5 \% \mathrm{OM}$. Avaliaram-se o número de brotos por ramo, a porcentagem de gemas brotadas e o número de ramos com broto, o número e a produção de frutos por planta. A antecipação da época de quebra de dormência provocou a diminuição do número de frutos produzidos na região centro-sul do Paraná. A produção de frutos foi diretamente proporcional à porcentagem de gemas brotadas no primeiro surto de brotação. A aplicação de cianamida hidrogenada promoveu antecipação da brotação e aumento na produção de frutos de caquizeiro; no entanto, a mistura de micronutrientes e óleo mineral não influenciou na brotação e produção de frutos de caquizeiro.

Termos para indexação: reguladores vegetais, Diospyrus kaki, brotação.

\section{TIME AND ACTIVE INGREDIENTS FOR BUD BREAK OF JAPANESE PERSIMMON CV. FUYU}

\begin{abstract}
This work aimed to rise fruit market period and search options to stimulate bud break, evaluating three dates and different active ingredients in bud break of persimmon 'Fuyu'. Mineral oil, hydrogen cyanamide $(\mathrm{CH})$, micro and macronutrients mixture were tested in three application dates in a factorial scheme with 5 treatments and 3 application dates. The traetments were: T1-control, T2- $0.5 \%$ OM, T3-: $2 \% \mathrm{CH}+$ $0.5 \%$ OM, T4- $1 \%$ micronutrients $+0.2 \%$ macronutrients $+0.5 \% \mathrm{OM}$; T5- $2 \%$ micronutrients $+0.2 \%$ macronutrients $+0.5 \%$ OM. It was analyzed the number of sprouts per branch, the sprouting percentage, the number of branches with sprouts, and the number and fruits production per plant. Bud break anticipation reduced fruits number in South Center region of Paraná. Fruit production was directly proportional to budburst in the first sprout cycle. $\mathrm{CH}$ application promotes budburst anticipation and raises persimmon fruits production, mineral oil and nutrients mixtures did not influence on budburst and production benefits.
\end{abstract}

Index Terms: plant regulators, Diospyrus kaki, budburst.

\section{INTRODUÇÃO}

A cultura do caqui vem crescendo no Brasil, com 8.322 ha plantados, produção de 164.849 t e produtividade de $19.839 \mathrm{~kg} / \mathrm{ha}$ (REETZ et al., 2007), principalmente nos estados do Sul. A produção está concentrada na cv. Fuyu, enquanto as outras cultivares apresentam projeção limitada. Entre esses cultivares destaca-se o 'Giombo', bastante cultivado no Paraná e importante por ser tardio. Colhido em maio, escapando do período de grande oferta no mercado (BLUM et al., 2008). Entretanto, existem vários entraves na comercialização, como a colheita muito concentrada, a falta de unidades armazenadoras e a baixa conservação frigorificada, que necessitam ser sanados para maior rentabilidade do produtor (FAGUNDES et al., 2006).

O período de dormência a que a planta está sujeita (FAQUIM et al., 2007) é outro fator limitante. Em estudos realizados por BERNARDES \& GODOY (1988), foi observado que, no Estado do Paraná, existe irregularidade anual na distribuição de horas de frio abaixo de $7,2^{\circ} \mathrm{C}$, influindo, negativamente, na brotação de gemas nos ramos de produção. Mes-

\footnotetext{
${ }^{1}$ (Trabalho 191-08). Recebido em: 22-07-2008. Aceito para publicação em: 19-11-2008.

${ }^{2}$ Eng. Agron. Prof. Dr. Do Departamento de Fitotecnia e Fitossanidade, UEPG, Av. Carlos Cavalcante, 4748, 84030-900 Ponta GrossaPR.E-mail: rayub@uepg.br

${ }^{3}$ Eng. Agron., MSc, Universidade Estadual de Ponta Grossa (UEPG), 84030-900 Ponta Grossa, PR. E-mail: juliusblum@yahoo.com.br. ${ }^{4}$ Eng. Agron. Dr. Pesquisador Instituto agronômico do Paraná (IAPAR), Av. Presidente Kennedy, s/n Rod. do Café, Km 496, 84001-970 - Ponta Grossa, PR. E-mail: marcelo_malgarim@iapar.br
} 
mo usando cultivares de baixa exigência em frio, a quebra de dormência é necessária para uniformizar e antecipar a brotação e compatibilizar o florescimento de cultivares de polinização cruzada, bem como antecipar e melhorar a produção nessas regiões (CRUZ JUNIOR \& AYUB, 2002). Nos caquizeiros, tanto em relação à época de quebra de dormência (BOTELHO et al., 2006; SOZIM et al., 2007), quanto aos produtos usados para a mesma (ROBERTO et al., 2006) precisam ser mais bem estudados. A cianamida hidrogenada $\left(\mathrm{H}_{2} \mathrm{CN}_{2}\right)$ é um produto eficiente na quebra de dormência do caquizeiro (MIZOBUTSI et al., 1997; 2003; 2004). No entanto, trata-se de um produto tóxico ao homem (SETTIMI et al., 2005), que pode ser fitotóxico e sem concorrentes no mercado, sendo limitante a produção integrada de frutos no Brasil (SANHUEZA et al., 2003). A busca por novas moléculas para solucionar este problema é constante, passando por produtos naturais, como o extrato de alho (BOTELHO \& MULLER, 2007) e várias formulações à base de macro e micronutrientes. A mistura de micronutrientes ( $\mathrm{S}, \mathrm{Zn}, \mathrm{Mo}, \mathrm{Co})$ formulada com Polihexose comercializada como Ubyfol Acordexplus (AC), somada à fertilizantes organominerais ( $\mathrm{N}, \mathrm{K}_{2} \mathrm{O}, \mathrm{C}$ orgânico), como, por exemplo, o produto comercial kymonplus (KY), têm sido comercializados com propósito de quebra de dormência em diversas fruteiras, no entanto não existem comprovações científicas da eficiência deste produto.

Com o objetivo de aumentar o período de oferta de frutos e buscar alternativas à falta de opções para estimular a brotação no caquizeiro 'Fuyu', avaliaram-se épocas e diferentes princípios ativos para a quebra de dormência.

\section{MATERIAL E MÉTODOS}

O experimento foi conduzido em pomar da cultivar Fuyu com 10 anos de idade, espaçamento de 5 metros entre linhas e 3 metros entre plantas, irrigado por microaspersão, localizado no município de Porto Amazonas-PR, (latitude 25 32 ' 22" S; longitude $49^{\circ} 54^{\prime} 44^{\prime \prime} \mathrm{W}, 876 \mathrm{~m}$ de altitude).

O delineamento experimental foi o de blocos ao acaso, em esquema fatorial 3 X 5, com 4 repetições. Sendo os fatores constituídos por 3 épocas de aplicação (20-07; 30-07 e 10-08-2007) e 5 tratamentos para quebra de dormência (T1- testemunha; T2- $0,5 \%$ de Óleo mineral (OM); T3- 1,0\% de CH (produto comercial Dormex) $+0,5 \%$ de óleo mineral (OM); T4- 1,0\% de AC (mistura de micronutrientes (S, Zn, Mo, Co) formulada com Polihexose, produto comercial Ubyfol Acordexplus) $+0,2 \%$ de KY (fertilizante organomineral $\left(\mathrm{N}, \mathrm{K}_{2} \mathrm{O}, \mathrm{C}\right.$ orgânico), produto comercial kymonplus) $+0,5 \%$ de OM; T5$2,0 \%$ de $\mathrm{AC}+0,2 \%$ de $\mathrm{KY}+0,5 \%$ de OM. A unidade experimental foi composta por três plantas, sendo as avaliações realizadas somente na planta central.

O número de brotos por ramo, a porcentagem de gemas brotadas e o número de ramos com broto foram obtidos a partir da contagem do total de gemas e do total de brotos em 20 ramos selecionados ao acaso por parcela, em duas épocas de avaliação (6-09 e 10-10-2007). Foram avaliados também o número e a produção de frutos por planta.

Todos os resultados foram submetidos à análise de variância para verificar a interação entre os fatores e as médias, comparadas pelo teste de Tukey, a $5 \%$ de probabilidade.

\section{RESULTADOS E DISCUSSÃO}

Não ocorreu interação significativa entre os fatores para nenhuma variável analisada, portanto os efeitos de tratamentos para a quebra de dormência e época de aplicação serão discutidos separadamente.

$\mathrm{O}$ uso de $\mathrm{CH}$ promoveu a antecipação da brotação nos caquizeiros. Na primeira avaliação realizada, aproximadamente $36 \%$ das gemas haviam brotado, e $90 \%$ dos ramos continham pelo menos uma gema brotada no tratamento com $\mathrm{CH}$, sendo que, nos demais tratamentos, apenas 1 a 3\% das gemas haviam brotado, e menos de $15 \%$ dos ramos continham pelo menos uma gema brotada (TABELA 1). Na $2^{\mathrm{a}}$ avaliação, não foram detectadas diferenças significativas quanto à porcentagem de gemas brotadas entre os tratamentos testados, revelando que, nessa data, já havia ocorrido naturalmente a quebra de dormência, sendo que aproximadamente $50 \%$ das gemas haviam brotado em todos os tratamentos. Portanto, a aplicação isolada de $\mathrm{OM}$, ou de $\mathrm{OM}$ com $\mathrm{AC}$ e KY, não influenciou na brotação do caquizeiro, visto que estes tratamentos não diferiram do tratamento-controle em nenhuma das três épocas estudadas.

$\mathrm{O}$ uso de $\mathrm{CH}$ também proporcionou o aumento do número de frutos por planta e, consequentemente, a produção de caquis (TABELA 1). Estes resultados concordam com os obtidos por MIZOBUTSI et al. (2003), trabalhando com caqui, em macieiras CRUZ JUNIOR \& AYUB (2002) e em kiwi SCHUCK \& PETRI (1995). A maior fixação de frutos à planta pela aplicação de CH foi atribuída por GEORGE \& NISSEN (1993) ao aumento da atividade metabólica no ovário, aumentando o fluxo de metabólitos para o fruto, diminuindo a queda do mesmo.

No entanto é importante notar que, na se- 
gunda avaliação (10-10-2007), onde havia cessado a brotação, não ocorreram diferenças do número de brotos por ramo e a porcentagem de gemas brotadas entre os tratamentos. Contudo, o tratamento com $\mathrm{CH}$ ocasionou aumento de, aproximadamente, $50 \%$ no número e produção de frutos. Isto demonstra que não é o número total de brotos que define o número de frutos e, consequentemente, a produção. Pois ocorreu correlação negativa entre o número de brotos por ramo e a produção de frutos (FIGURA 1) e porcentagem de gemas brotadas na segunda avaliação com a produção de frutos (FIGURA 2). Portanto, a hipótese de que tenha ocorrido uso excessivo de energia da planta para a produção de ramos, em detrimento da produção de frutos, pode ser vislumbrada. No presente estudo, o que parece ter contribuído para o aumento da produção, foi a antecipação da brotação, visto que ocorreu correlação positiva entre a porcentagem de gemas brotadas na primeira avaliação e a produção de frutos (FIGURA 3). O aumento na produção de frutos devido à antecipação do início da brotação também foi verificada por MIZOBUTSI et al. (2003).

O número de ramos com broto foi avaliado somente na primeira época de amostragem, pois, na avaliação de 10-10-2007, todos os ramos possuíam pelo menos um broto, não havendo, portanto, necessidade de demonstração dos resultados.

A antecipação na época de aplicação dos tratamentos para quebra de dormência não apresentou efeito na antecipação da brotação, pois, na primeira época de avaliação, não ocorreram diferenças significativas quanto ao percentual de gemas brotadas nas diferentes épocas em que foram aplicados os tratamentos (TABELA 2), divergindo dos resultados obtidos por MIZOBUTSI et al. (1997), que verifica- ram que a antecipação da aplicação de $\mathrm{CH}$ resultou na antecipação da brotação, porém em condições ambientais diferentes do presente estudo.

A aplicação dos tratamentos na primeira época proporcionou o maior aumento na porcentagem de gemas brotadas na segunda época de avaliação e, também, o aumento no número total de brotos por ramo. No entanto, ocasionou também a diminuição do número de frutos produzidos, que pode ter ocorrido em função da vegetação excessiva da planta. Ao contrário do observado neste trabalho, MIZOBUTSI et al. (2003) observaram que, quanto mais antecipada foi a aplicação da $\mathrm{H}_{2} \mathrm{CN}_{2}$, melhor foi a produção de frutos. No entanto, deve-se considerar que as condições climáticas entre as regiões onde foram realizados os trabalhos, são distintas, principalmente no que diz respeito às temperaturas mínimas, pois, logo após a primeira época de aplicação, ocorreram temperaturas próximas e abaixo de $0^{\circ} \mathrm{C}$. Em outro trabalho realizado por MIZOBUTSI et al. (2004), foram obtidos resultados semelhantes ao deste, onde as aplicações antecipadas de $\mathrm{H}_{2} \mathrm{CN}_{2}$ diminuíram a fixação de frutos, mas, desta vez, devido ao déficit hídrico. No entanto, no presente trabalho, essa hipótese deve ser descartada, visto que o pomar foi irrigado.

A porcentagem de gemas brotadas na primeira avaliação, o número de brotos por ramo, o número de frutos por planta e a produção de frutos foram semelhantes quando os tratamentos foram aplicados em 30-07 e 10-08-2007. E a porcentagem final de gemas brotadas foi menor, quanto mais tardia a aplicação dos tratamentos.

TABELA 1 - Parâmetros vegetativos e de produção de caqui 'Fuyu' submetido a diferentes tratamentos para a quebra de dormência. Cada valor é a média de três épocas de quebra de dormência. Porto Amazonas-PR, 2007.

\begin{tabular}{lccccc}
\hline \multirow{2}{*}{ Tratamento } & $\begin{array}{c}\mathrm{N}^{\circ} \text { de ramos } \\
\text { com broto } \\
06-09-2007\end{array}$ & $\begin{array}{c}\% \text { de gemas brotadas por } \\
\text { ramo }\end{array}$ & $\begin{array}{c}\mathrm{N}^{\circ} \text { de } \\
\text { frutos/ } \\
\text { planta }\end{array}$ & $\begin{array}{c}\text { Produção/ } \\
\text { planta } \\
\text { 06 }\end{array}$ \\
\hline Testemunha & $15,0 \mathrm{~B}$ & $3,1 \mathrm{~B}$ & $53,6 \mathrm{~A}$ & $102,5 \mathrm{~B}$ & $19, \mathrm{~B}$ \\
$0,5 \% \mathrm{OM}$ & $7,08 \mathrm{~B}$ & $1,3 \mathrm{~B}$ & $53,0 \mathrm{~A}$ & $109,3 \mathrm{~B}$ & $20,3 \mathrm{~B}$ \\
$\mathrm{CH} 2 \%+0,5 \% \mathrm{OM}$ & $90,8 \mathrm{~A}$ & $36,9 \mathrm{~A}$ & $50,3 \mathrm{~A}$ & $172,5 \mathrm{~A}$ & $32,9 \mathrm{~A}$ \\
$1 \% \mathrm{AC}+0,2 \% \mathrm{KY}+0,5 \% \mathrm{OM}$ & $4,17 \mathrm{~B}$ & $1,0 \mathrm{~B}$ & $53,7 \mathrm{~A}$ & $98,4 \mathrm{~B}$ & $19,0 \mathrm{~B}$ \\
$2 \% \mathrm{AC}+0,2 \% \mathrm{KY}+0,5 \% \mathrm{OM}$ & $12,9 \mathrm{~B}$ & $2,6 \mathrm{~B}$ & $51,1 \mathrm{~A}$ & $94,2 \mathrm{~B}$ & $17,6 \mathrm{~B}$ \\
\hline $\mathrm{CV}(\%)$ & 56,3 & 54,3 & 12,1 & 37,1 & 35,8 \\
\hline
\end{tabular}

Médias seguidas da mesma letra na coluna não diferem entre si, pelo teste de Tukey, a 5\% de probabilidade.

$\mathrm{CH}$ - Cianamida Hidrogenada (Dormex);

OM - Óleo Mineral;

$\mathrm{AC}$ - micronutrientes (Ubifol Acordexplus);

$\mathrm{KY}$ - fertilizante organomineral (Kymonplus). 
TABELA 2 - Parâmetros vegetativos e de produção de caqui 'Fuyu' submetido a três épocas para a quebra de dormência. Cada valor é média dos cinco tratamentos para a quebra de dormência. Porto Amazonas-PR, 2007.

\begin{tabular}{|c|c|c|c|c|}
\hline Época de aplicação & $\begin{array}{l}\mathrm{N}^{\circ} \text { de brotos/ } \\
\text { ramo } \\
10-10-2007\end{array}$ & $\begin{array}{l}\% \text { de gemas brotadas por } \\
\text { ramo } \\
6-09-2007 \quad 10-10-2007\end{array}$ & $\begin{array}{l}\mathrm{N}^{\circ} \text { de frutos/ } \\
\text { planta }\end{array}$ & $\begin{array}{c}\text { Produção por } \\
\text { planta } \\
\text { kg }\end{array}$ \\
\hline $\begin{array}{l}20-07-07 \\
30-07-07 \\
10-08-07\end{array}$ & $\begin{array}{l}7,4 \mathrm{~A} \\
5,8 \mathrm{~B} \\
5,5 \mathrm{~B}\end{array}$ & $\begin{array}{ll}9,3 \mathrm{~A} & 62,8 \mathrm{~A} \\
9,9 \mathrm{~A} & 49,6 \mathrm{~B} \\
7,7 \mathrm{~A} & 44,7 \mathrm{C}\end{array}$ & $\begin{array}{l}92,9 \mathrm{~B} \\
126,7 \mathrm{~A} \\
126,7 \mathrm{~A}\end{array}$ & $\begin{array}{l}18,2 \mathrm{~A} \\
23,1 \mathrm{~A} \\
24,1 \mathrm{~A}\end{array}$ \\
\hline CV (\%) & 14,0 & 54,3 & 37,1 & 35,8 \\
\hline
\end{tabular}

Médias seguidas da mesma letra na coluna não diferem entre si, pelo teste de Tukey, a 5\% de probabilidade.

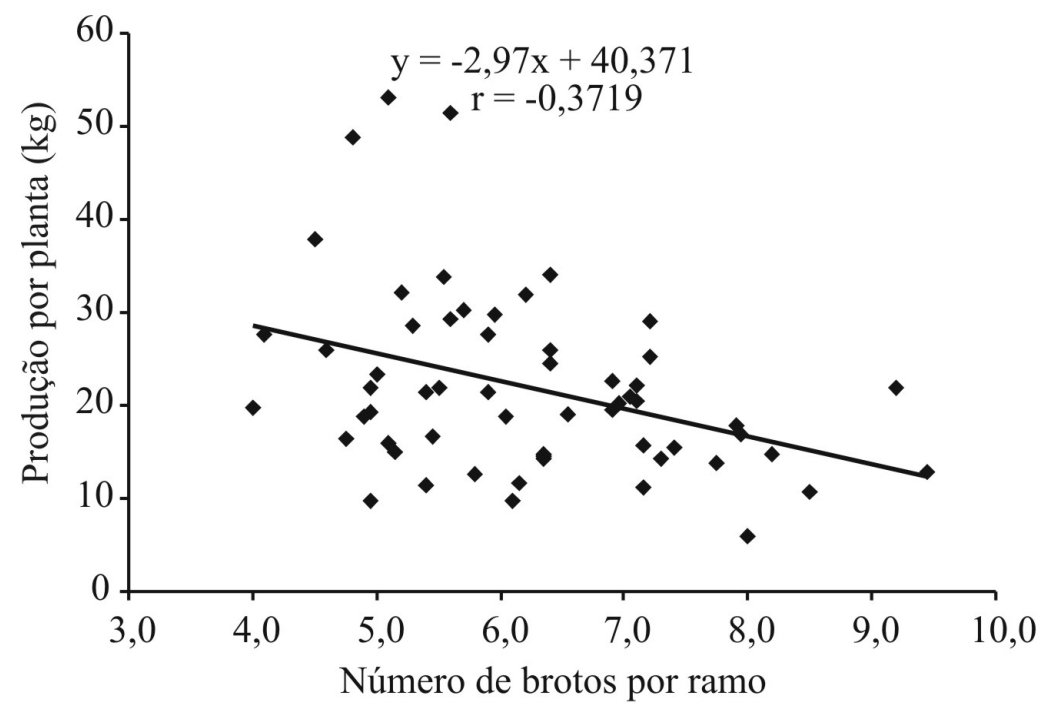

FIGURA 1- Correlação entre o número de brotos por ramo e a produção por planta. Porto Amazonas - PR, 2008.

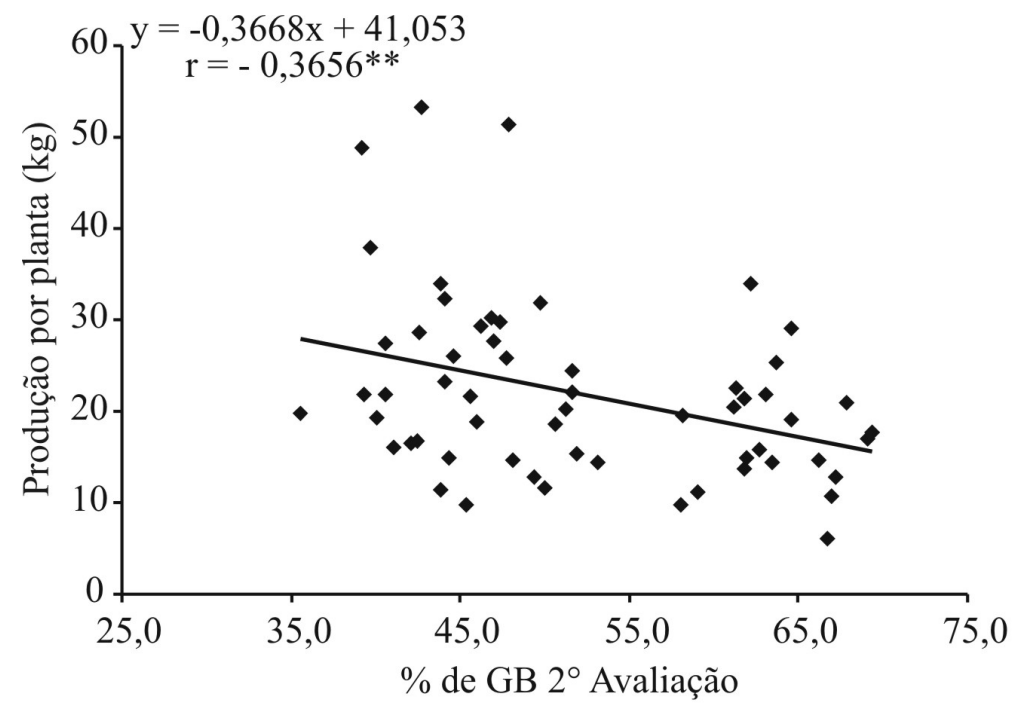

FIGURA 2- Correlação entre a \% de gemas brotadas (GB) e a produção por planta em 10-10-2007, data da $2^{\circ}$ avaliação. Porto Amazonas - PR, 2008. 


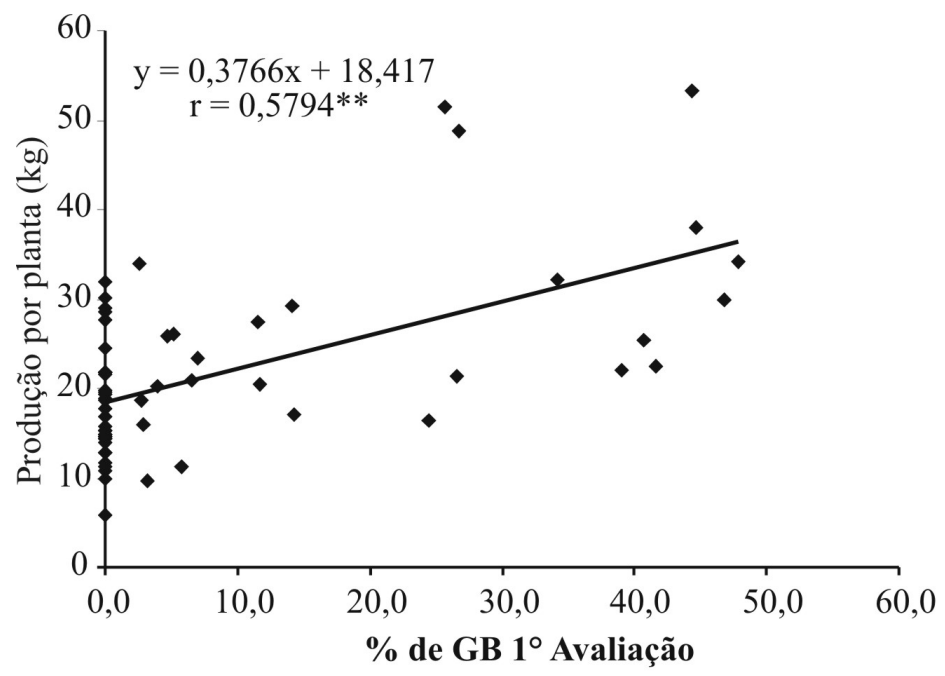

FIGURA 3- Correlação entre a \% de gemas brotadas (GB) e a produção por planta em 06-09-2007, data da $1^{\mathrm{a}}$ avaliação. Porto Amazonas - PR, 2008.

\section{CONCLUSÕES}

1-A aplicação de cianamida hidrogenada promoveu antecipação da brotação e aumento na produção de frutos de caqui, porém não deve ser feita antes do final de julho, devido aos riscos de geada na região. $\mathrm{E}$ a $\mathrm{CH}$ foi o único produto capaz de quebrar a dormência do caquizeiro.

2-A produção de frutos foi diretamente proporcional à porcentagem de gemas brotadas no primeiro surto de brotação, evidenciando que o segundo surto desvia as reservas dos frutos para um excesso de vegetação.

\section{REFERÊNCIAS}

BERNARDES, L. M.; GODOY, H. A cultura da macieira no Paraná. Circular IAPAR, Londrina, n. 50, p. 11-18, 1988.

BLUM, J.; HOFFMANN, F. B.; AYUB, R. A.; PRADO, P. V. B.; MALGARIM, M. B. Destanização do caqui "Giombo" com etanol e ethephon. Revista Ceres, Viçosa, v. 5 n. 1, p. 54-59, 2008.

BOTELHO, R. V.; AYUB, R. A.; MULLER, M. M. L. Somátoria de horas de frio e de unidades de frio em diferentes regiões do estado do Paraná. Scientia Agrária, Curitiba, v.7, p. 89-96, 2006.
BOTELHO, R. V.; MULLER, M. M. L. Extrato de alho como alternativa na quebra de dormência de gemas em macieiras cv. Fuji Kiko. Revista Brasileira de Fruticultura, Jaboticabal, v. 29, n. 1, p. 37-41, 2007.

CRUZ JÚNIOR, A. de O.; AYUB, R. A. Quebra de dormência de gemas de macieira cv. Eva tratadas com cianamida hidrogenada. Revista Brasileira de Fruticultura, Jaboticabal, v. 24, n. 2, p. 576578, 2002.

FAGUNDES, A. F.; DABUL, A. N. G.; AYUB, R. A.; Aminoethoxivinilglicina no controle do amadurecimento de frutos de caqui cv. Fuyu. Revista Brasileira de Fruticultura, Jaboticabal, v. 28, n.1 p. 73-75, 2006.

FAQUIM, R.; SILVA, I. D. da; CARVALHO, R. I. N. de. Necessidade de frio para quebra de dormência de gemas de caquizeiro 'Fuyu'. Revista Brasileira de Fruticultura, Jaboticabal, v. 29, n.3 p. 438-444, 2007.

GEORGE, A. P.; NISSEN, R. J. Effects of growth regulants on defoliation, flowering, and fruit maturity of the low chill peach cultivar Flordaprince in subtropical Australia. Australian Journal of Experimental Agriculture, Collingwood, v.33, p.787-795, 1993. 
MIZOBUTSI, G. P.; BRUCKNER, C. H.; SALOMÃO, L. C. C.; NEVES, J. C. L.. Antecipação da colheita de caqui 'Costata' através da quebra de dormência com cianamida hidrogenada e óleo mineral. Revista Brasileira de Fruticultura, Jaboticabal, v.26, no.2, p.209-211. 2004.

MIZOBUTSI, G. P.; BRUCKNER, C. H.; SALOMÃO, L. C. C.; RIBEIRO, R. A.; MOTTA, W. F. Efeito da aplicação de cianamida hidrogenada e de óleo mineral em caquizeiro. Revista Brasileira de Fruticultura, Jaboticabal, v.25, n.1, p.89-92. 2003.

MIZOBUTSI, G. P.; BRUCKNER, C.H.; SALOMÃO, L.C.C.; NEVES, J.C.L. Efeito da aplicação de cianamida hidrogenada e óleo mineral em sete variedades de caquizeiro Diospyros kaki L. Revista Ceres, Viçosa, v.44 /255, p. 547-556, 1997.

REETZ, E. R. Anuário brasileiro da fruticultura. Santa Cruz do Sul: Ed. Gazeta, 2007. 136p.

ROBERTO, S. R.; KAGUEYAMA, M. H.; SANTOS, C. E. Indução de brotação da macieira 'Eva'em região de baixa incidência de frio. Revista Brasileira de Fruticultura, Jaboticabal, v. 28, n.1 p. 128-130, 2006.
SANHUEZA, R. M. V.; ANDRIGUETO, J. R.; KOSOSKI, A. R. Situação atual da produção integrada de frutas no Brasil. In: SEMINARIO BRASILEIRO DE PRODUÇÃO INTEGRADA DE FRUTAS, 5., 2003. Bento Gonçalves. Anais... Bento Gonçalves: Embrapa-CNPUV, 2003. p. 23-25.

SCHUCK, E.; PETRI, J.L. The effect of concentrations and application of hydrogen cyanamide on kiwifruit dormancy breaking. Acta Horticulturae, Wageningen, v.395, p. 177-183, 1995.

SETTIMI, L.; DAVANZO, F.; FARAONI M. G.; RICHMOND, D.; CALVERT, G. M. Update: Hidrogen cyanamide-related ilnesses-Italy, 2002-2004.

Morbidity and Mortality Weekly Report, Atlanta, v.54, p. 405-408, 2005.

SOZIM, M.; FERREIRA, F. P.; AYUB, R. A.; BOTELHO, R. V. Época de poda e quebra de dormência em videiras cv. Niagara Rosada. Semina, Londrina, v. 28, p. 201-206, 2007. 